\title{
Management of Immune-Mediated Paraneoplastic Neurological Disorders
}

\section{(ㄷ) (1) $($ 우 $\Theta$}

Authors

Ilya Ayzenberg, Ralf Gold, Ingo Kleiter

Affiliation
Department of Neurology, St. Josef Hospital, Ruhr University
Bochum, Germany
Key words
paraneoplastic, autoantibody, encephalitis, polyneuropathy
Bibliography
DOI https://doi.org/10.1055/s-0043-112730
Neurology International Open 2017; 1: E264-E274
C Georg Thieme Verlag KG Stuttgart · New York
ISSN $2511-1795$
Correspondence
Dr. Ilya Ayzenberg
Klinik für Neurologie St. Josef Hospital Ruhr-Universität
Bochum
Gudrunstr. 56
44791 Bochum
Germany
ilya.ayzenberg@rub.de

\section{Introduction}

Paraneoplastic neurological syndromes (PNS) are immune-mediated paraneoplastic disorders of the nervous system caused by tumor-induced disturbance of immune tolerance to neuronal antigens. Tumors expressing neuronal proteins, either intracellularly or on cell membranes, are most relevant ( $\vee$ Table 1 ). The detection of antineuronal antibodies (Abs) confirms the diagnosis of PNS in patients presenting with typical clinical signs and symptoms of the syndrome; however, approximately $18 \%$ of cases remain seronegative [1]. The immune response can be directed against structures of the central or peripheral nervous system, neuromuscular synapses or muscles. PNS may present with a variety of signs and symptoms, making it difficult to reliably establish the diagnosis [2]. Some general clinical features are common and support the suspicion of PNS:

\begin{abstract}
Paraneoplastic neurological disorders are rare and clinically heterogeneous diseases. They can affect both the central and peripheral nervous system as well as the neuromuscular junction and muscle. Neurological deficits develop in 2/3 of cases prior to cancer diagnosis. The diagnostic approach includes screening for antineuronal antibodies and a search for the underlying tumor. A prompt tumor therapy in combination with immunotherapy is the cornerstone in the management of these diseases. Due to lack of clinical trials, treatment recommendations are based on case series and expert opinions. Highdose corticosteroids, intravenous immunoglobulins and apheresis therapies are often used in the acute stage of the disease. These therapies should be started as early as possible, e. g., during tumor screening, in order to prevent irreversible damage. Long-term treatment is mostly immunosuppressive and depends on the specific paraneoplastic syndrome. Outcomes vary depending upon the prognosis of the underlying cancer and the nature of the antineuronal antibodies. Disorders with antibodies directed against antigens on the neuronal cell surface are highly sensitive to $B$ cell-directed therapies and mostly associated with a favorable outcome. A thorough review of published data on actual treatment recommendation is provided along with discussion of currently not validated, but potentially effective new therapies.
\end{abstract}

- age > 45 years (few exceptions, e. g., anti-NMDAR encephalitis);

- subacute initial manifestation, followed by progression of symptoms over weeks or months;

- multilocular symptoms (several structures of the central and peripheral nervous system can be affected simultaneously);

- cranial MRI findings can demonstrate symmetrical lesions, e. g., in the cerebellum or temporal lobe, with initial contrast enhancement and atrophy in later stages, or may be unremarkable (especially in the early stage);

- inflammatory changes in the cerebrospinal fluid (CSF): isolated intrathecal immunoglobulin synthesis or identical oligoclonal bands in CSF and serum, moderate lymphocytic pleocytosis $(<100 / \mu l)$, signs of blood-brain barrier disruption.

Whether the Abs are directed against intracellular antigens or surface antigens is the most important factor determining the clinical 
- Table 1 Paraneoplastic antibodies: associated neurological syndromes and tumors.

\section{Target antigen}

Clinical presentation\#

Obligate paraneoplastic Abs (directed against intracellular antigens; tumor association >95\%)

Hu (ANNA-1) Polyneuropathy (mostly sensory), encephalomyelitis, limbic encephalitis, opsoclonus-myoclonus syndrome

Yo (PCA-1)

Ri (ANNA-2)

CV2 (CRMP5)

PNMA $1 / 2$

Cerebellar degeneration

Amphiphysin

Cerebellar degeneration, opsoclonus-myoclonus syndrome

Encephalomyelitis, sensory neuropathy,

Limbic encephalitis, brainstem encephalitis, potentially with hypothalamic involvement

$\operatorname{Tr}(\text { DNER })^{2}$

PCA2

ANNA-3

Facultative paraneoplastic Abs (tumor association, \%)

1. Abs to synaptic receptors

$\operatorname{NMDAR}(20-50 \%)$

Anti-NMDAR encephalitis

AMPAR $(65 \%)$

$\mathrm{GABA}_{B} R(50 \%)$

$\mathrm{GABA}_{A} \mathrm{R}(5 \%)$

mGluR5 (70\%)

mGluR1 (10\%)

$\alpha$-GlyR (20\%)

Stiff-person syndrome, encephalopathy, myelopathy, cerebellar degeneration, opsoclonus-myoclonus syndrome

Cerebellar degeneration

Encephalitis, LEMS, polyneuropathy

Polyneuropathy, cerebellar degeneration, limbic encephalitis

Limbic encephalitis

Limbic encephalitis

Encephalitis

Encephalitis

Cerebellar degeneration

PERM, stiff-person syndrome

2. Abs to ion channels and other surface proteins

LGI1 (5-10\%)

Caspr2 (20\%)

$\operatorname{DPPX}(<10 \%)$

$\operatorname{VGCC}(50-70 \%)$

Limbic encephalitis

Limbic encephalitis, Morvan's syndrome

Encephalitis

LEMS

3. Abs to intracellular antigens

$\operatorname{GAD}(<10 \%)$

Stiff-person syndrome, limbic encephalitis, cerebellar degeneration

\section{Association with tumor}

SCLC, rarely thymoma, prostate cancer, neuroblastoma in children

Ovarian cancer or breast cancer

Breast cancer or ovarian cancer, SCLC, NSCLC

SCLC, thymoma

Men < 45 years: mostly germ cell tumor; women, men $>45$ years: NSCLC, breast cancer

Breast cancer, SCLC

Lymphoma

$S C L C>N S C L C$

$S C L C$

Ovarian teratoma (age: $12-45$ years), carcinomas (rare, mostly $>45$ years)

SCLC, thymoma, breast cancer

SCLC

Thymoma

Hodgkin's lymphoma

Hodgkin's lymphoma

Thymoma, lymphoma, breast cancer

Thymoma

Thymoma

Lymphoma

SCLC » prostate cancer, thymoma, lymphoma

NET, breast cancer, thymoma

\# rare or not fully characterized associations between Abs and clinical syndromes are not listed

2 transmembrane protein

The following Abs are not included in the table:

1. Abs associated with retinopathy, myasthenia gravis and myositis

2. Non-paraneoplastic Abs

3. Abs not clinically characterized to a sufficient degree or Abs only described in a few cases

Abbreviations: AMPAR, $\alpha$-amino-3-hydroxy-5-methyl-4-isoxazolepropionic acid receptor; ANNA, antineuronal antibody; Caspr2, contactin-associated protein-related 2; CRMP5, collapsin-response mediator protein 5; DNER, Delta/Notch-like epidermal growth factor-related receptor; DPPX, dipeptidyl-peptidase-like protein-6; GABAR, gamma-aminobutyric acid receptor; GAD65, glutamate decarboxylase (65 kDa); $\alpha$-GlyR, glycine receptor alpha 1; LGI1, leucine-rich glioma inactivated 1; mGluR, metabotropic glutamate receptor; NET, neuroendocrine tumors; NMDA, N-methyl-D-aspartate receptor; (N)SCLC, (non)-small cell lung cancer; PCA, Purkinje cell cytoplasmic antibody; PNMA, paraneoplastic Ma antigen; VGCC, voltage-gated calcium channel; ZIC, zinc finger transcription factor

course, treatment response and prognosis of PNS [2,3]. Depending on tumor association, obligate and facultative paraneoplastic Abs are differentiated (Tab. 1).

Clinically, the following PNS exist $[3,4]$ :

A. In the central nervous system:

- Encephalomyelitis (incl. brainstem encephalitis)
- Limbic encephalitis

- Subacute cerebellar degeneration

- Opsoclonus-myoclonus syndrome

- Optic neuritis/neuromyelitis optica

- Myelopathy/myelitis

- Stiff person syndrome 
B. In the peripheral nervous system, neuromuscular junction and the muscle:

- Paraneoplastic polyneuropathies (incl. subacute sensory neuronopathy, distal symmetric sensorimotor neuropathy, polyradiculoneuropathy)

- (Pan)dysautonomia (incl. chronic intestinal pseudo-obstruction)

- Neuromyotonia

- Myasthenia gravis

- Lambert-Eaton syndrome

- Myositis

In this review we discuss the diagnosis and management of PNS. With regard to facultative paraneoplastic syndromes which are more frequently of autoimmune origin, such as neuromyelitis optica or myasthenia gravis, the reader should refer to pertinent reviews $[5,6]$.

\section{Diagnostic and Pathogenetic Significance of Antibodies to Intracellular and Surface Antigens}

Antibodies (Abs) to intracellular antigens or so-called "classical" Abs are almost always tumor-associated (exception: GAD-Abs). They can be associated with various tumors and a variety of neurological conditions [7]. However, a direct pathogenetic significance of these Abs has not yet been demonstrated. Abs to intracellular antigens serve as useful diagnostic markers in the targeted tumor search in PNS. Here, cell-mediated immune responses play a major pathogenetic role, typically resulting in tissue damage within a few weeks. The neuropathological picture is characterized by infiltration of cytotoxic CD8 ${ }^{+} \mathrm{T}$ cells which are frequently co-localized with neurons and induce apoptosis by releasing perforin and granzyme B [8]. The compartmentalization of the inflammatory reaction in the central and peripheral nervous system frequently limits the efficacy of immunotherapies in PNS with Abs to intracellular antigens, resulting in an overall unfavorable prognosis.

By contrast, Abs to surface antigens are not obligatory paraneoplastic and, especially in young patients, occur often as a primary autoimmune disorders. These Abs are targeted against receptors, channels or associated membrane proteins and typically are of direct pathogenetic significance. Accordingly, the target antigen determines the characteristic clinical presentation, for example, short faciobrachial dystonic seizures in patients with LGI1-Abs or neuromyotonia in patients with CASPR2-Abs. Dysfunction of the targeted cell surface proteins is frequently reversible and neuronal or synaptic loss are less severe compared to PNS with Abs against intracellular antigens. Immunotherapies, especially those depleting Abs, e.g., plasma exchange or immunoadsorption, can bring dramatic improvement. Patients with Abs to extracellular antigens have a considerably better prognosis compared to those with Abs to intracellular antigens.

\section{General Principles of Treatment}

Given that paraneoplastic disorders are rare conditions and that the range of $A b$-associated syndromes continues to broaden, controlled studies are not available for the majority of PNS. Most treat- ment recommendations are based on case series and expert opinions. Considering the potentially irreversible damage, the treatment of PNS should be started as early as possible.

Main principles of PNS management include:

1. Detection and resection of the tumor in order to remove the source of peripheral antigen stimulation;

2. Rapid start of immunosuppressive treatment;

3. Symptomatic treatment.

Prompt and aggressive tumor treatment is the most relevant therapeutic strategy in PNS.

\section{Tumor detection and efficacy of tumor therapy}

In about two thirds of patients with PNS, the tumor is still unknown at the time of onset of neurological symptoms [1]. Since the PNS-causing immune response slows down cancer growth, the tumors can be relatively small and difficult to diagnose at the time of initial neurological manifestation. The presence of multiple Abs in about one third of patients with PNS helps to focus the diagnostic workup on specific tumors [7,9]. PNS are most frequently associated with lung cancer (small cell » non-small cell lung cancer), ovarial carcinoma and teratoma, breast cancer, Hodgkin's disease and non-Hodgkin lymphoma, thymoma, and prostate cancer $[1,10]$. It is also important to rule out testicular tumors in young men and neuroblastoma in young children. Based on the target antigen and the suspected tumor, a targeted stepwise diagnostic approach is recommended ( $\triangleright$ Table 2 ) [11]. Should the standard workup yield negative results, it can be supplemented by a whole-body FDG PET/ CT scan. While being an expensive investigation, it offers the advantage of improving the diagnostic sensitivity [12]. The limitations of FDG PET/CT include false-positive findings in the presence of inflammatory processes and false-negative findings with tumors exhibiting low proliferation rates (e. g., differentiated teratomas) or non-metastatic skin cancers. If no tumor can be detected, the diagnostic workup should be repeated in 3 months' time and then every 6 months for a period of at least 4 years [11]. Adequate tumor treatment frequently results in the stabilization or even improvement of the neurological deficits [13-15]. Close interdisciplinary collaboration is crucial, since tumor resection, especially in combination with chemotherapy and/or radiotherapy, may be sufficient to control the PNS.

\section{Principles of immunotherapy}

Immunotherapy should be started as early as possible, preferably within 4 weeks of initial manifestation and before the neurological deficits have reached a plateau and become irreversible [16-18]. If the suspicion of PNS is clearly supported by clinical evidence, immunotherapy may be started even without definite tumor diagnosis (exception: suspected lymphoma).

Acute treatment is usually based on either corticosteroid pulse therapy (e. g., $5 \times 1000 \mathrm{mg}$ methylprednisolone IV, if required with oral tapering) or intravenous immunoglobulin G (IVIG, e. g., $0.4 \mathrm{~g} /$ $\mathrm{kg}$ bodyweight for 5 days). Should the patient not improve with these regimes, treatment escalation to an apheresis therapy (plasma exchange or immunoadsorption) can be undertaken. Basically, IVIG treatment and apheresis therapies are more effective in patients with Abs directed against surface antigens compared to in- 
- Table 2 Recommendations for stepwise cancer screening in patients with paraneoplastic syndromes $[1,11]$.

Tumor, prevalence ${ }^{\#}$

Lung cancer SCLC $38.4 \%$, NSCLC $7.9 \%$

Ovarian cancer $10.5 \%$

Ovarian teratoma ${ }^{\#}$

Breast cancer $9.7 \%$

Hodgkin's disease 3.0\%, non-Hodgkin's lymphoma 3.4\%

Thymoma $2.7 \%$

Testicular tumors $1.7 \%$

\section{Stepwise diagnosis (sensitivity of test method)}

Chest CT scan (80-85\%) $\rightarrow$ FDG PET(CT) $\rightarrow$ bronchoscopy / EB-US with biopsy

Transvaginal US (69-90\%), Ca125 (62\%) $\rightarrow$ pelvic/abdominal CT scan $\rightarrow$ FDG PET (CT)

Transvaginal US (58-94\%) $\rightarrow$ pelvic/abdominal CT/MRI (93-96\%) $\rightarrow$

$\rightarrow$ chest CT scan, if required (extrapelvic teratomas)

Mammography, US $\rightarrow$ breast MRI (71-100\%), FDG PET (CT), if required

Chest/abdomen CT scan, US $\rightarrow$ FDG PET (CT)

Chest CT/MRI (75-90\%) $\rightarrow$ FDG PET (CT)

US $(72 \%), A F P+\beta-H C G \rightarrow$ pelvic $C T / M R I *$, biopsy in the case of microcalcifications

\# Prevalence among all PNSs, based on Euronetwork Database; no data on ovarian teratoma available; Abbreviations: AFP, alpha fetoprotein; $\beta$-HCG, beta human chorionic gonadotropin; CA125, cancer antigen 125; EB-US, endobronchial ultrasound; US, ultrasound

tracellular antigens with mainly T cell-mediated immune responses $[3,16,19,20]$. If the patient does no improve with acute treatment, an early (typically up to 2 weeks after the above-mentioned primary treatment) escalation to cyclophosphamide (as a shortterm high-dose treatment with $750-1000 \mathrm{mg} / \mathrm{m}^{2} \mathrm{IV}$ ) or rituximab (e. g., $500 \mathrm{mg}$ IV on days 1 and 15) can be undertaken [16, 21, 22]. Here, it is important to ensure that the patient receives appropriate concomitant medications, such as uromitexan along with cyclophosphamide or steroids and antihistamines along with rituximab.

Long-term treatment is typically based on classical oral immunosuppressants, such as azathioprine $(2-3 \mathrm{mg} / \mathrm{kg} / \mathrm{d})$, mycophenolate mofetil (250-1000 mg b.i.d.) or oral cyclophosphamide $(1-2 \mathrm{mg} / \mathrm{kg} / \mathrm{d})$ as a monotherapy or in combination with oral steroids. Alternatively, rituximab (e. g., every 6 months) or IVIG at regular intervals (usually every 4 to 8 weeks) may be effective as well.

\section{Special immunotherapeutic and symptomatic treatment strategies for selected PNS}

Paraneoplastic Cerebellar Degeneration (PCD)

In the majority of patients with PCD, Abs to intracellular antigens (Yo, Hu, Ri, Tr, Ma2, CV2/CRMP5, Amphiphysin, GAD, Zic4, ANNA3, PCA-2; sporadically, Homer3, CARPVIII, PKCy, Ca/ARHGAP26) are detected, while Abs to surface antigens (VGCC, in combination with Lambert-Eaton myasthenic syndrome (LEMS); sporadically mGluR1) are rare [23]. The clinical picture is dominated by progressive cerebellar ataxia, reaching a plateau within the first weeks to months in the majority of patients, with $50-70 \%$ of patients becoming wheelchair-bound during this time [24]. In most cases, irreversible loss of Purkinje cells is the underlying cause of this condition. The prognosis of patients with Abs to intracellular antigens is particularly unfavorable (especially, if Hu- or Yo-, CV2/CRMP5Abs are present) [14]. In some patients, stabilization or slight improvement can be achieved with early tumor resection, followed by chemotherapy, where indicated [16]. While subsequent immunotherapies, including corticosteroids, IVIG, plasma exchange, cyclophosphamide and tacrolimus, generally have little effect, individual cases with relevant treatment response have been reported [16, 25-27]. Patients with Tr-Abs after successful lymphoma treat- ment and patients with Ri-Abs under immunotherapy have a more favorable prognosis [28, 29]. Overall, response to immunotherapy is better in patients with GAD-Abs; these Abs are rarely tumor-associated [30]. Steroid pulse therapy and IVIG, plasma exchange, and rituximab may be effective in these patients. In patients with VGCC-Abs, PCD may occur alone or in combination with LEMS. In LEMS, the response rate is higher compared with PCD and cerebellar symptoms stabilize in up to $40 \%$ of patients after tumor therapy and immunotherapy [31].

In patients with cerebellar tremor, symptomatic treatment with propranolol (30-180 mg/d), primidone $(30-500 \mathrm{mg} / \mathrm{d})$, topiramate (25-100 mg/d), or clonazepam (1.5-6 mg/d) can be attempted [32].

\section{Encephalomyelitis and limbic encephalitis (EM, LE)}

Apart from so-called "classical” EM and LE (associated with Abs against $\mathrm{Hu}$, Ma, GAD, CV2/CRMP5), this disease spectrum has been substantially expended over the last decade, especially with the identification of several Abs to surface antigens (NMDAR, VGKC, DPPX, AMPAR, GABAbR, dopamine receptor 2, mGluR5).

Patients with Abs to surface antigens have a much better prognosis, except for cases with additional classical paraneoplastic Abs $[33,34]$. LE is most frequently associated with NMDAR-, LGI1- and Caspr2-Abs; treatment of these forms of encephalitis will be discussed individually. There are significantly less data available on LE associated with other Abs to surface antigens.

Principles of therapy of LE with Abs to intracellular antigens are similar to those of classical PNS (as described above). The prognosis remains poor with the exception of Ma2-Ab-associated encephalomyelitis in which approx. $30 \%$ of patients experience improvement after adequate tumor treatment and immunotherapy [35].

\section{A. Anti-NMDA receptor encephalitis (anti-NMDAR encephalitis)}

Anti-NMDAR encephalitis is the most common type of antibody-mediated encephalitis and accounts for approx. $4 \%$ of all encephalitis cases [36]. Patients with anti-NMDAR encephalitis typically do not experience relevant neuronal loss, but reversible dysfunction caused by internalization of NMDA receptors from the cell surface $[37,38]$. This mechanism explains the good outcome, up 
to complete recovery, in about $75 \%$ of patients after adequate therapy [39]. The acute stage of the disease can last for several months and patients frequently undergo intensive care unit treatment during this time. Mild cognitive deficits may persist [40]. Mortality, often caused by intensive care complications, is about 7-9\%. Of all anti-NMDAR encephalitis cases, $38 \%$ are paraneoplastic and associated in young women, very rarely in men, with teratomas, and in patients $>45$ years with carcinomas [41]. The occurrence of teratomas increases among adolescents and is at $15 \%$ in female patients $<14$ years, at $30 \%<18$ years and at $60 \%>18$ years [39]. Thus, immediate targeted tumor screening is crucial in patients with anti-NMDAR encephalitis. Since FDG PET-CT is not sensitive enough to detect well-differentiated teratomas, a negative scan result is considered inconclusive. Instead it is recommended to perform an abdominal MRI scan and a thorough gynecological examination, including endovaginal ultrasound. In children $<12$ years and in men, anti-NMDAR encephalitis of paraneoplastic etiology is much rarer.

So far, treatment efficacy data of 4 larger retrospective case series have been published [42-45]. Any tumor detected has to be immediately removed. Up to $80 \%$ of patients improve with tumor resection in combination with first-line immunotherapy: steroid pulse therapy, IVIG or plasma exchange [39]. However, in non-paraneoplastic cases, first-line immunotherapy results in improvement in only approx. $50 \%$ of patients $[39,44]$. Controlled studies comparing the above-mentioned first-line immunotherapies are not available; however, a small series $(n=9)$ found an advantage for early plasma exchange treatment [46]. Given the direct pathogenetic role of NMDAR-Abs, the use of apheresis therapy is very reasonable. The efficacy of immunoadsorption seems to be similar to those of plasma exchange $[47,48]$. As expected, an early immunotherapy ( $<40$ days after initial manifestation) is associated with a significantly better prognosis [43]. If no tumor can be detected or the response to treatment is delayed, second-line therapy with rituximab or cyclophosphamide should be considered [49]. Again, there is a lack of comparative studies. Cyclophosphamide crosses the blood-brain barrier, but is associated with toxic effects and has a negative impact on fertility which is highly relevant in young women. For this reason, rituximab continues to be increasingly used in clinical practice. In approx. $75 \%$ of cases, second-line therapy results in further significant improvement of the symptoms $[39,42,44,46]$. In several cases and case series successful administration of other immunosuppressants, including azathioprine, methotrexate and mycophenolate mofetil, has been reported [49]. However due to lack of systemic studies a definite judgment on the efficacy of these drugs cannot be given. In individual refractory pediatric cases, intrathecal administration of methotrexate and methylprednisolone was effective [50]. Of special interest, and reasonable from a pathogenetic perspective, is escalation therapy with the proteasome inhibitor bortezomib [51, 52]. Bortezomib results in a targeted reduction of plasma cells and consequently Abs production. In two case series, female patients with refractory anti-NMDAR encephalitis showed a significant clinical improvement after the administration of bortezomib and a rapid decrease in serum NMDAR-Ab-titers [51, 52].

The recurrence rate of anti-NMDAR encephalitis is in the range of $12-25 \%$; it is higher in patients with non-paraneoplastic disease compared with patients after teratoma resection $[42,44]$. In these cases, treatment with a second-line therapy for a limited period of time (e. g., 2 years) is recommended, even in patients who showed acceptable initial improvement. Relapses can occur several months to years after the first episode of the disease; second-line therapies were demonstrated to be effective [44]. The reason why some patients relapse remains unclear. The postulated phenomenon of epitope spreading has not been confirmed in patients with several recurrences or poor prognosis [53]. Intra-individual correlation between Ab-titer changes (especially in the CSF) and prognosis or development of a recurrence was reported [53]. A characteristic feature of the monophasic course is the rapid decline in anti-NMDAR-Abtiters in serum and CSF under treatment $[43,53]$. Inter-individually, Ab-titers are higher in patients with teratomas and in patients with non-paraneoplastic anti-NMDAR encephalitis with poor prognosis $[42,43,53]$.

\section{B. Anti-VGKC antibody encephalitis (anti-VGKC encephalitis)}

Voltage-gated potassium channels (VGKC) are expressed both in the central nervous system and juxtaparanodally on peripheral motor nerves. In anti-VGKC encephalitis, autoantibodies are not directed against potassium channels, but against three VGKC-associated proteins: leucine-rich glioma inactivated-1 (LGI1), contactin-associated protein-like-2 (CASPR2) and contactin-2 [54-56]. The majority of patients (60-80\%) are men, mainly in the second half of life. LGI1-Abs are typically associated with limbic encephalitis, hyponatremia, and in some cases with faciobrachial dystonic seizures. The latter are characteristic of LGI1 encephalitis, last only seconds ( $<3 \mathrm{~s}$ ) and occur many times a day [57]. At a later stage of disease, patients may develop generalized seizures. Anti-VGKC encephalitis of paraneoplastic origin is rather rare $(0-11 \%)[54,55]$. Apart from encephalitis, CASPR2-Abs can cause neuromyotonia (Isaak-Merten's syndrome) which is characterized by peripheral motor nerve hyperexcitability. The combination of encephalitis, neuromyotonia with autonomous involvement, and insomnia is referred to as Morvan's syndrome. It is associated with tumors in approx. $20 \%$ of cases [58]. It is unknown whether contactin-2-Abs play an independent pathogenetic role, since they usually occur in combination with anti-LGI1 or anti-CASPR2. The significance of other anti-VGKC-Abs (LGI1 and CASPR2 negative) also remains unclear. A recent study found that $38 \%$ of these Abs are directed against intracellular epitopes of the Kv1 subunit of the VGKC complex and are presumably of no clinical significance [59].

Overall, patients with anti-VGKC encephalitis have a favorable prognosis: approx. $75 \%$ of patients respond to immunotherapy $[58,60]$. However, in contrast to anti-NMDAR encephalitis, neurodegenerative changes occur in addition to functional disturbances (the latter probably due to reduced AMPA receptor expression) [61]. Especially in patients with anti-LGl1-Abs, memory impairment and other cognitive deficits may persist [62]. The few available neuropathological findings show immunoglobulin deposits, partially with complement activation and moderate lymphocytic infiltration, as well as neuronal loss in the hippocampus and amygdala [8].

In most cases, symptomatic treatment with anticonvulsants alone is insufficient. In patients with anti-VGKC encephalitis of paraneoplastic origin, treatment of the underlying tumor is crucial. Regarding immunotherapy, either corticosteroids (IV or orally), IVIC 
or plasma exchange may be used. There are no prospective comparative studies available. However, some findings indicate that combined and earlier (within the first 2 months after initial manifestation) treatment is associated with a better prognosis $[49,63]$. A retrospective study $(n=14)$ found that combination therapy of IVIG plus steroids was superior to steroid monotherapy [64]. In an open prospective study, all patients $(n=9)$ improved when treated with the combination of plasma exchange, IVIG and steroid pulse therapy followed by oral steroid treatment [65].

An immediate response to treatment is rarely observed; the majority of patients improve after several weeks of treatment [66]. In most cases, first a reduction in seizure frequency is noted, while cognitive deficits tend to respond much later [60]. Other immunotherapeutic agents, such as azathioprine, tacrolimus, mycophenolate mofetil and rituximab may be tried in refractory cases [49]. Sometimes patients experience relapses, correlating with an increase in Ab-titers [67].

\section{Other types of encephalitis associated with antibodies to surface proteins}

Antibodies to DPPX (dipeptidyl-peptidase-like protein 6) were detected in patients with multifocal central nervous system deficits and dysautonomia. Rarely, they are associated with lymphomas. In a retrospective case series, immunotherapy (corticosteroids, IVIG, PLEX alone or combined with rituximab or cyclophosphamide) resulted in improvements in about two thirds of patients [68]. Due to the limited data available, no conclusions can be drawn regarding the efficacy of the various treatments.

Encephalitis with AMPAR-Abs (alpha-amino-3-hydroxy-5-methyl-4-isoxazolepropionic acid receptor) is tumor-associated in about two thirds of cases and characterized by an aggressive course of disease [69]. Tumor resection in combination with immunotherapy (corticosteroids or IVIG) can lead to clinical improvement. Relapses are frequent and occur unrelated to tumor recurrence. In most cases, relapses can be prevented by treatment with rituximab and cyclophosphamide [34].

Anti-GABAbR-Abs typically cause limbic encephalitis and less often ataxia. In $50 \%$ of cases a tumor (frequently small cell lung cancer) is detected [70]. The combination of tumor resection, chemotherapy and immunotherapy can be effective (particularly in case of a favorable tumor prognosis) [33].

Encephalitis associated with anti-IgLON5-Abs is a very rare disease of unknown origin. Patients usually do not respond to immunotherapy, even though IgLON5 is a cell surface protein [71]. Neuropathology demonstrates a tauopathy involving the midbrain tegmentum and the hypothalamus. Apart from brain stem and motor symptoms, parasomnia is a typical feature of the disease.

Opsoclonus-myoclonus syndrome (OMS)

The disease occurs in small children and adults. In contrast to other classical PNS, OMS may follow a chronic relapsing course. Young children (<5 years) with OMS are typically seronegative for autoantibodies (except for a few cases with anti-Hu-Abs) and in approx. $50 \%$ of these patients OMS is associated with neuroblastoma [72]. The outcomes achieved with the standard treatments (tumor therapy, corticosteroids or ACTH in pediatric patients, often combined with IVIG; if required, plasma exchange) are often unsatisfactory and associated with residual deficits [73]. More aggressive therapeutic strategies, such as rituximab and cyclophosphamide, can improve long-term prognosis, including cognitive and behavioral deficits [74, 75].

Among adults, OMS is in approx. $40 \%$ of paraneoplastic origin and in $60 \%$ idiopathic or presumably of parainfectious etiology (e. g., associated with HIV, mumps, CMV, EBV) [76]. In older patients ( $>50$ years) with paraneoplastic OMS, lung cancer or breast cancer (typically with anti-Ri-Abs) are frequently detected. Other antibodies to intracellular antigens ( $\mathrm{Hu}, \mathrm{Ma} 2$, amphiphysin, CV2/ CRMP5) are less often detected and Abs to surface antigens (GABAbR, NMDAR, DPPX, and $\alpha$-GlyR [rather unspecific]) have been described in isolated cases only. Often adult OMS remains seronegative as well. Following effective tumor treatment, neurological symptoms may improve [77]. The role of immunotherapy in patients with paraneoplastic OMS remains unclear. Reports of an effective treatment with corticosteroids, cyclophosphamide, or plasma exchange are limited to few cases [73]. In younger patients, an association between teratoma, anti-NMDAR-Abs negative encephalitis (mostly with brain stem involvement) and OMS has been described [78]. After tumor resection and immunotherapy (methylprednisolone, in some cases combined with IVIG and plasma exchange), 8 of 10 patients became symptom-free and 2 experienced residual ataxia and dysarthria. Likewise, patients with parainfectious OMS responded well to immunotherapy (e. g., IVIG) and overall had a better prognosis $[77,79]$. Clonazepam $(3 \times 0.5-2 \mathrm{mg} / \mathrm{d})$ or propranolol $(3 \times 40-80 \mathrm{mg} / \mathrm{d})$ can be used for symptomatic treatment.

\section{Stiff-person syndrome (SPS)}

In patients with SPS, antibodies to the synaptic vesicle-associated protein amphiphysin (approx. 2-10\% of cases, mostly with breast cancer), the intracellular enzyme glutamic acid decarboxylase (GAD65, approx. 60-80\%, facultative paraneoplastic), or the cell surface-associated glycine receptor $\alpha 1$ subunit (GlyR, approx. $10 \%$, facultative paraneoplastic) are detected [80-82]. The hallmark of SPS is generalized muscle rigidity with painful muscle spasms, an exaggerated startle response and a characteristic gait abnormality with freezing of gait and hyperlordosis. Symptoms can also be localized and affect one limb only (stiff-limb syndrome). Apart from classical SPS, anti-GlyR-Abs may occur in patients with so-called PERM syndrome (progressive encephalomyelitis with rigidity and myoclonus). Signs of brainstem involvement, primarily eye movement abnormalities, ataxia, as well as convulsive seizures and autonomic dysregulation, can occur in patients with PERM syndrome. In contrast to other PNS, most patients with SPS experience a significant improvement with symptomatic treatment alone, which therefore is used as first-line therapy. GABA-A receptor agonists such as diazepam (average dose $40 \mathrm{mg} / \mathrm{d}$ ) or clonazepam $(1-6 \mathrm{mg} / \mathrm{d}$ ) and GABA-B receptor agonists such as baclofen (average dose $60 \mathrm{mg} / \mathrm{d}$ ) are in many cases effective in reducing muscle spasm and rigor; however, tolerance may develop later [81]. In individual cases, positive effects have been reported for treatment with levetiracetam, valproate or gabapentin [83-85]. In extremely severe cases, intrathecal baclofen treatment can be used [86]. Besides symptomatic treatment, a long-term immunosuppressive therapy is usually required. In patients with paraneoplastic SPS (es- 
pecially breast cancer with amphiphysin-Abs) cancer treatment, including chemotherapy, has the highest priority. With regard to immunotherapy, both corticosteroids and immunosuppressants (e. g., cyclophosphamide) can be effective $[80,87,88]$. Remarkably, experimental data has indicated that anti-amphiphysin-Abs may play a direct pathogenetic role in SPS; up to now, this has not been demonstrated for any other antibody directed against an intracellular antigen [89]. In SPS patients with anti-GAD-Abs, IVIG ( $2 \mathrm{~g} / \mathrm{kg}$ per cycle every 4 weeks) was found to be effective and is recommended as first-line therapy $[81,90]$. Alternatively, steroid pulse therapy (e. g., methylprednisolone $500 \mathrm{mg}$ IV for 5 days) with subsequent switch to oral prednisone, gradually tapered off to a maintenance doses of $5-10 \mathrm{mg} / \mathrm{d}$, is recommended [91]. However, the use of steroids is limited in many cases by diabetes, a common comorbidity, necessitating treatment with steroid-saving medications, such as mycophenolate mofetil, azathioprine, or cyclophosphamide. Treatment escalation to apheresis therapy can be effective, regardless of the type of antibody. In SPS patients who tested positive for anti-GAD-Abs, plasma exchange treatment achieved a significant improvement of symptoms in approx. half of the patients, even in some patients who did not respond to firstline therapy [92]. While rituximab was reportedly effective in individual cases, a smaller blinded study failed to show a convincing effect of rituximab $[81,93]$. As a general rule, treatment of SPS should be started as early as possible, before the patient develops irreversible tissue damage. According to a large retrospective study ( $n=99$ ), relevant improvement of symptoms can be achieved during the first year after initial manifestation only [81].

Patients testing positive for anti-glycine receptor $\alpha 1$-Abs generally have a better prognosis and respond well to immunotherapy [82]. Similar to anti-NMDAR encephalitis, internalization of the glycine receptor is discussed as the underlying pathogenetic mechanism. Relapses occur in approx. $10 \%$ of cases [82]. A lethal outcome is rare and may occur in rapidly developing courses with autonomic involvement during the acute stage of disease. For most cases, successful treatment with steroid therapy (initially as a pulse therapy, with later switch to oral prednisone) in combination with plasma exchange and/or IVIG has been reported.

\section{Paraneoplastic myelopathy (PM)}

Paraneoplastic myelopathy or myelitis is a rare disease which may remain unrecognized until late in the course of the disease. Clinically, PM presents as a subacute or slowly progressive myelopathy which may initially be misdiagnosed as a seronegative NMOSD or PPMS [94]. PM is usually associated with anti-amphiphysin- or anti-CV2/CRMP5-Abs. Less frequently, other antibodies directed against intracellular antigens (Hu, Ri, Yo, Ma1/2, GAD, PCA2, ANNA3) or surface antigens (VGCC, VGKC, glycine receptor $\alpha 1$ subunit) are detected. In rare cases, especially in elderly patients, anti-AQP4-Abs may be induced by a tumor, presenting a paraneoplastic form of neuromyelitis optica spectrum disorder [95, 96]. No clinical studies evaluating the efficacy of PM treatments are available. The prognosis is unfavorable in the majority of cases. In a cases series, transient improvement was achieved in 8 of 26 patients, but only 3 patients experienced long-lasting independent mobility [94]. In individual cases, corticosteroids or cyclophosphamide were effective $[97,98]$.

\section{Paraneoplastic polyneuropathy (pPNP)}

The disease often precedes clinical tumor manifestation [73]. Onconeural Abs can be detected in $80 \%$ of patients, primarily anti-Hu(frequently associated with lung cancer) or anti-CV2/CRMP5-Abs (with lung cancer, thymoma, or neuroendocrine tumors). Peripheral involvement in combination with the corresponding characteristic central PNS may occur along with anti-amphiphysin-, anti-Yo-, anti-Ri-, anti-Tr-, or anti-PCA2-Abs [99]. Damage of peripheral sensory ganglia results in subacute sensory, often asymmetric polyneuropathy with pain, paresthesia and sensory ataxia (classical subacute sensory polyneuropathy). Patients with autonomic involvement develop orthostatic hypotension, impotence, anhidrosis, and sicca syndrome. Sensorimotor polyneuropathy may occur as well. Electroneurography helps to reveal axonal and demyelinating variants. CSF analysis frequently shows moderately increased protein levels and intrathecal immunoglobulin $G$ synthesis, so that the patient may be misdiagnosed as having CIDP. Generally, immunotherapy has little effect in patients with pPNP [17]. In a large study with anti-Hu-associated paraneoplastic encephalomyelitis and polyneuropathy $(n=200)$, tumor therapy was the only treatment associated with stabilization of the neurological deficits [13]. Neuropathic pain can be treated with pregabalin $(75-600 \mathrm{mg} / \mathrm{d})$, gabapentin $(300-3600 \mathrm{mg} / \mathrm{d})$ or carbamazepine $(200-1200 \mathrm{mg} / \mathrm{d})$, if required in combination with amitriptyline (25-75 mg/d). Apart from classical onconeural antibodies, polyneuropathic syndromes occur with VGCC-Abs, VGKC-Abs and Abs to ganglionic nAChRs. Ganglionic nAChR-Abs are in $30 \%$ of paraneoplastic origin and can cause both dysautonomia and polyneuropathy [100]. Combined tumor treatment and immunotherapy (e. g., prednisone alone or plus azathioprine) can improve dysautonomia. In non-paraneoplastic cases, initial IVIG therapy, if required with further escalation to plasma exchange, and immunosuppression with azathioprine or mycophenolate mofetil can be effective [101].

\section{Lambert-Eaton myasthenic syndrome (LEMS)}

LEMS is associated with lung cancer and antibodies to P/Q-type VGCC in $50-60 \%$ and $85 \%$ of cases, respectively [102]. Furthermore, LEMS can be triggered by prostate cancer or thymoma. While LEMS is usually tumor-associated in male patients $>40$ years, it is typically of autoimmune origin in younger female patients. Patients testing positive for anti-SOX1-Abs usually have lung cancer (95\% specificity). The presynaptic dysfunction caused by anti-VGCC-Abs results in a reduced release of acetylcholine at the motor endplate and muscarinic synapses of the autonomic nervous system. From this, the classical LEMS symptoms can be derived: generalized muscle weakness (often more pronounced in the proximal legs), reduced tendon reflexes, fatigue, and autonomic changes (dry mouth, constipation, impotence, etc.).

Management of paraneoplastic LEMS is based on tumor therapy, including tumor resection and chemotherapy, which may also have an immunosuppressant effect. Symptomatic therapy alone can improve neuromuscular transmission at a clinically relevant level. 3,4-diaminopyridine augments the release of acetylcholine und can be gradually increased (WARNING: side effects such as epileptic seizures and arrhythmias $)$ up to the target dose $(4 \times 10 \mathrm{mg} / \mathrm{d}$ $-5 \times 20 \mathrm{mg} / \mathrm{d})$ [103]. In refractory cases pyridostigmine $(3 \times 30 \mathrm{mg} /$ 
d-6 $\times 60 \mathrm{mg} / \mathrm{d}$ ), a drug with post-synaptic action, can be added to the treatment regimen. If response to this treatment is inadequate, prednisone can be used in combination with azathioprine; in severe cases, escalation to repeated IVIG therapy or plasma exchange is an option [102]. The prognosis of tumor-associated LEMS is significantly poorer than that of the autoimmune type.

\section{Paraneoplastic myositis}

Apart from dermatomyositis, which is in $30 \%$ of cases of paraneoplastic origin, polymyositis and necrotizing myositis can occur in tumor patients. According to a recent meta-analysis, factors associated with a paraneoplastic etiology of myositis include advanced age, male gender, skin necrosis, and dysphagia [104]. Detection of anti-155/140-Abs in cases with negative classical Abs (anti-jo-1, anti-PM-Scl, anti-U1-RNP, anti-U3-RNP, anti-Ku) is in 94\% associated with a paraneoplastic origin [105]. While anti-synthetase-Abs are typically associated with autoimmune etiology, their detection does not reliably rule out a paraneoplastic origin of the disease [106]. Dermatomyositis occurs most frequently with ovarian cancer, breast cancer, lung cancer, pancreatic cancer, stomach cancer or colorectal cancer, while polymyositis is associated with lung cancer, bladder cancer or lymphoma [107]. Assumingly, the immunological cross-reaction between undifferentiated myoblasts and tumor cells is triggered by the expression of identical antigens [108]. The courses of the both diseases are linked: while tumor recurrence is frequently associated with deterioration of the muscle disease, effective tumor treatment results in an improvement of muscle strength [109].

The immunotherapy of first choice are glucocorticosteroids (initially $1-2 \mathrm{mg} / \mathrm{kg}$ for 2-4 weeks; alternatively, in severe cases initially as a pulse therapy (500 mg IV for 3-5 days, followed by gradual oral tapering). For relapse prevention, long-term treatment for 1-3 years with low-dose prednisone in combination with azathioprine $(2-3 \mathrm{mg} / \mathrm{kg})$ or methotrexate $(7.5-25 \mathrm{mg} /$ week $)$ is recommended [110]. If response to these treatments is inadequate, IVIG ( $1-2 \mathrm{~g} / \mathrm{kg} /$ cycle every $6-8$ weeks) can be added. For refractory cases, rituximab $(2 \times 1000 \mathrm{mg}$ IV at intervals of 2 weeks), mycophenolate mofetil $(2 \mathrm{~g} / \mathrm{d}$ ) or cyclophosphamide $(1-2 \mathrm{mg} / \mathrm{kg} / \mathrm{d}$ orally or $0.5-1.0 \mathrm{~g} / \mathrm{m}^{2}$ body surface area i. v. per treatment cycle) should be taken into consideration. The differential diagnosis of patients deteriorating under long-term treatment includes steroid myopathy; thus, an attempt to reduce the steroid dose should be made. If electromyography shows abnormal spontaneous activity, steroid myopathy is rather unlikely.

\section{Outlook}

The most significant advances of recent years have been achieved in the treatment of PNS with antibodies to surface antigens. B cell-depleting therapy with rituximab has gained in importance. While rituximab can reduce short-lived plasma cells and immunoglobulin G4 synthesis, it has no effect on long-lived plasma cells [111]. In patients intolerant to rituximab, further humanized antiCD20-Abs, such as ocrelizumab and ofatumumab, may become an alternative in the future. Long-lived plasma cells can be influenced by two other approaches. The proteasome inhibitor bortezomib reduces both subtypes of plasma cells. This drug has been approved for the treatment of multiple myeloma and mantle cell lymphoma. By inhibiting proteolysis, bortezomib disrupts several signal cascades, ultimately resulting in cell death. These effects are most relevant in plasma cells actively producing immunoglobulins and lead to a rapid reduction in Abs synthesis in patients with autoimmune diseases [112]. Bortezomib was effective in several refractory cases of anti-NMDAR encephalitis [51,52]. Likewise, the use of tocilizumab, a monoclonal antibody targeting the interleukin-6 (IL-6) receptor, may be an interesting alternative in some refractory cases of PNS. Blocking IL6 receptors results in reduction of Ab production by plasma cells and in the differentiation of Th17 cells [113]. Apart from rheumatic disease, tocilizumab has been used successfully to treat several Ab-induced neurological disorders, including NMOSD and Caspr2-Ab-positive limbic encephalitis [114-116].

By contrast, no relevant breakthrough has so far been achieved in the immunotherapy of T-cell-mediated paraneoplastic syndromes. One of the potential options discussed is tacrolimus, a calcineurin inhibitor. It inhibits the activation of T cells, crosses the blood-brain barrier and its side effect profile is superior to that of cyclosporine. Tacrolimus was partially effective in combination with steroid therapy in some patients with classical PNS [117].

Another potentially relevant and important trend is the increasing use of immune checkpoint inhibitors (e. g., the anti-CTLA-4 Ab ipilimumab and the anti-PD-1 Abs nivolumab and pembrolizumab) in tumor therapy. Apart from an augmented immune response to tumor cells this treatment can also cause immune-mediated paraneoplastic syndromes $[118,119]$.

\section{Summary and Conclusions for Clinical Practice}

1. A broad serological work-up, comprising serum and, ideally, CSF testing, plays an important role in the diagnosis of PNS. Nevertheless, both seronegative cases and incidental low-titer findings without corresponding clinical signs are frequently found.

2. Prospective studies evaluating treatments for PNS are not available. Systematic data collection is of utmost importance (e.g., in Germany integrated in the GENERATE network).

3. Tumor screening (including whole-body FDG PET/CT scan, if necessary) and tumor therapy should be performed as early as possible. In many cases, systemic tumor therapy is also an effective treatment of PNS.

4. First-line immunotherapies (steroid pulse therapy, IVIG, apheresis therapies) should be started without delay, even before tumor diagnosis, where necessary.

5. Immunotherapy can lead to dramatic improvement in PNS with antibodies to surface antigens. As a long-term therapy, rituximab is increasingly used.

6. In PNS with antibodies to intracellular antigens and a predominantly T-cell-mediated immune response, immunotherapy is of limited efficacy. Here, there is a need for new therapeutic strategies.

7. Immune checkpoint inhibitors used to treat cancer may induce the development of PNS. 
I. Ayzenberg received honoraria for consultancy from Roche and grant support from Chugai, none related to this manuskript. I. Kleiter received honoraria for consultancy or speaking from Bayer, Biogen, Chugai, Merck, Roche and Shire and grant support from Affectis, Chugai and Diamed, none related to this manuskript. R. Gold received speaker's honoraria from Baxter, Bayer Schering, Biogen Idec, CLB Behring, Genzyme, Merck Serono, Novartis, Stendhal, Talecris, TEVA. His department received grant support from Bayer Schering, Biogen Idex, Genzyme, Merck Serono, Novartis and TEVA.

\section{References}

[1] Giometto B, Grisold W, Vitaliani R et al. Paraneoplastic neurologic syndrome in the PNS Euronetwork database: A European study from 20 centers. Arch Neurol 2010; 67: 330-335

[2] McKeon A. Paraneoplastic and other autoimmune disorders of the central nervous system. Neurohospitalist 2013; 3: 53-64

[3] Leypoldt F, Wandinger K-P. Paraneoplastic neurological syndromes. Clin Exp Immunol 2014; 175: 336-348

[4] Gozzard P, Maddison P. Which antibody and which cancer in which paraneoplastic syndromes? Pract Neurol 2010; 10: 260-270

[5] Trebst C, Jarius S, Berthele A et al. Update on the diagnosis and treatment of neuromyelitis optica: Recommendations of the Neuromyelitis Optica Study Group (NEMOS). J Neurol 2014; 261: $1-16$

[6] Melzer N, Ruck T, Fuhr P et al. Clinical features, pathogenesis, and treatment of myasthenia gravis: A supplement to the Guidelines of the German Neurological Society. J Neurol 2016; 263: 1473-1494

[7] Pittock SJ, Kryzer TJ, Lennon VA. Paraneoplastic antibodies coexist and predict cancer, not neurological syndrome. Ann Neurol 2004; 56: 715-719

[8] Bauer ], Bien CG. Neuropathology of autoimmune encephalitides. Handb Clin Neurol 2016; 133: 107-120

[9] Horta ES, Lennon VA, Lachance DH et al. Neural autoantibody clusters aid diagnosis of cancer. Clin Cancer Res 2014; 20: 3862-3869

[10] Darnell RB, Posner JB. Paraneoplastic syndromes affecting the nervous system. Semin Oncol 2006; 33: 270-298

[11] Titulaer M], Soffietti R, Dalmau J et al. Screening for tumours in paraneoplastic syndromes: Report of an EFNS Task Force. Eur J Neurol 2011; 18: 19-e3

[12] Hadjivassiliou M, Alder S], Van Beek EJR et al. PET scan in clinically suspected paraneoplastic neurological syndromes: A 6-year prospective study in a regional neuroscience unit. Acta Neurol Scand 2009; 119: 186-193

[13] Graus F, Keime-Guibert F, Reñe R et al. Anti-Hu-associated paraneoplastic encephalomyelitis: Analysis of 200 patients. Brain J Neurol 2001; 124: 1138-1148

[14] Shams'ili S, Grefkens J, de Leeuw B et al. Paraneoplastic cerebellar degeneration associated with antineuronal antibodies: Analysis of 50 patients. Brain J Neurol 2003; 126: 1409-1418

[15] Candler PM, Hart PE, Barnett M et al. A follow up study of patients with paraneoplastic neurological disease in the United Kingdom. J Neurol Neurosurg Psychiatry 2004; 75: 1411-1415

[16] Vernino S, O’Neill BP, Marks RS et al. Immunomodulatory treatment trial for paraneoplastic neurological disorders. Neuro Oncol 2004; 6: $55-62$
[17] Keime-Guibert F, Graus F, Fleury A et al. Treatment of paraneoplastic neurological syndromes with antineuronal antibodies (Anti-Hu, anti-Yo) with a combination of immunoglobulins, cyclophosphamide, and methylprednisolone. J Neurol Neurosurg Psychiatry 2000; 68: 479-482

[18] Widdess-Walsh P, Tavee JO, Schuele S et al. Response to intravenous immunoglobulin in anti-Yo associated paraneoplastic cerebellar degeneration: Case report and review of the literature. J Neurooncol 2003; 63: 187-190

[19] Graus F, Vega F, Delattre JY et al. Plasmapheresis and antineoplastic treatment in CNS paraneoplastic syndromes with antineuronal autoantibodies. Neurology 1992; 42: 536-540

[20] Uchuya M, Graus F, Vega F et al. Intravenous immunoglobulin treatment in paraneoplastic neurological syndromes with antineuronal autoantibodies. J Neurol Neurosurg Psychiatry 1996; 60: 388-392

[21] Stark E, Wurster U, Patzold U et al. Immunological and clinical response to immunosuppressive treatment in paraneoplastic cerebellar degeneration. Arch Neurol 1995; 52: 814-818

[22] Shams'ili S, de Beukelaar J, Gratama JW et al. An uncontrolled trial of rituximab for antibody associated paraneoplastic neurological syndromes. J Neurol 2006; 253: 16-20

[23] Jarius S, Wildemann B. 'Medusa-head ataxia': The expanding spectrum of Purkinje cell antibodies in autoimmune cerebellar ataxia. Part 1: Anti-mGluR1, anti-Homer-3, anti-Sj/ITPR1 and anti-CARP VIII. J Neuroinflammation 2015; 12: 166

[24] Jones AL, Flanagan EP, Pittock S] et al. Responses to and Outcomes of Treatment of Autoimmune Cerebellar Ataxia in Adults. JAMA Neurol 2015; 72: 1304-1312

[25] Peterson K, Rosenblum MK, Kotanides $\mathrm{H}$ et al. Paraneoplastic cerebellar degeneration. I. A clinical analysis of 55 anti-Yo antibody-positive patients. Neurology 1992; 42: 1931-1937

[26] Rojas I, Graus F, Keime-Guibert F et al. Long-term clinical outcome of paraneoplastic cerebellar degeneration and anti-Yo antibodies. Neurology 2000; 55: 713-715

[27] Phuphanich S, Brock C. Neurologic improvement after high-dose intravenous immunoglobulin therapy in patients with paraneoplastic cerebellar degeneration associated with anti-Purkinje cell antibody. J Neurooncol 2007; 81: 67-69

[28] Briani C, Vitaliani R, Grisold W et al. Spectrum of paraneoplastic disease associated with lymphoma. Neurology 2011; 76: 705-710

[29] Greenlee JE. Treatment of paraneoplastic cerebellar degeneration. Curr Treat Options Neurol 2013; 15: 185-200

[30] Ariño H, Höftberger R, Gresa-Arribas N et al. Paraneoplastic neurological syndromes and glutamic acid decarboxylase antibodies. JAMA Neurol 2015; 72: 874-881

[31] Graus F, Lang B, Pozo-Rosich P et al. P/Q type calcium-channel antibodies in paraneoplastic cerebellar degeneration with lung cancer. Neurology 2002; 59: 764-766

[32] Tremor. Leitlinie der Deutschen Gesellschaft für Neurologie.Im Internet www.dgn.org Stand: Sep. 2012

[33] Höftberger R, Titulaer M], Sabater L et al. Encephalitis and GABAB receptor antibodies: Novel findings in a new case series of 20 patients. Neurology 2013; 81: 1500-1506

[34] Höftberger R, van Sonderen A, Leypoldt F et al. Encephalitis and AMPA receptor antibodies: Novel findings in a case series of 22 patients. Neurology 2015; 84: 2403-2412

[35] Dalmau J, Graus F, Villarejo A et al. Clinical analysis of anti-Ma2-associated encephalitis. Brain J Neurol 2004; 127: 1831-1844

[36] Granerod J, Ambrose HE, Davies NW et al. Causes of encephalitis and differences in their clinical presentations in England: A multicentre, population-based prospective study. Lancet Infect Dis 2010; 10: 835-844 
[37] Hughes EG, Peng X, Gleichman A] et al. Cellular and synaptic mechanisms of anti-NMDA receptor encephalitis. J Neurosci 2010; 30: 5866-5875

[38] Moscato EH, Peng X, Jain A et al. Acute mechanisms underlying antibody effects in anti-N-methyl-D-aspartate receptor encephalitis. Ann Neurol 2014; 76: 108-119

[39] Dalmau J, Lancaster E, Martinez-Hernandez E et al. Clinical experience and laboratory investigations in patients with anti-NMDAR encephalitis. Lancet Neurol 2011; 10: 63-74

[40] Finke C, Kopp UA, Prüss $\mathrm{H}$ et al. Cognitive deficits following anti-NMDA receptor encephalitis. J Neurol Neurosurg Psychiatry 2012; 83: 195-198

[41] Titulaer M], McCracken L, Gabilondo I et al. Late-onset anti-NMDA receptor encephalitis. Neurology 2013; 81: 1058-1063

[42] Dalmau J, Gleichman A], Hughes EG et al. Anti-NMDA-receptor encephalitis: Case series and analysis of the effects of antibodies. Lancet Neurol 2008; 7: 1091-1098

[43] Irani SR, Bera K, Waters P et al. N-methyl-D-aspartate antibody encephalitis: Temporal progression of clinical and paraclinical observations in a predominantly non-paraneoplastic disorder of both sexes. Brain J Neurol 2010; 133: 1655-1667

[44] Titulaer M], McCracken L, Gabilondo I et al. Treatment and prognostic factors for long-term outcome in patients with anti-NMDA receptor encephalitis: An observational cohort study. Lancet Neurol 2013; 12: $157-165$

[45] Viaccoz A, Desestret V, Ducray F et al. Clinical specificities of adult male patients with NMDA receptor antibodies encephalitis. Neurology 2014; 82: 556-563

[46] Pham HP, Daniel-Johnson JA, Stotler BA et al. Therapeutic plasma exchange for the treatment of anti-NMDA receptor encephalitis. J Clin Apheresis 2011; 26: 320-325

[47] Heine J, Ly L-T, Lieker I et al. Immunoadsorption or plasma exchange in the treatment of autoimmune encephalitis: A pilot study. J Neurol 2016; 263: 2395-2402

[48] Dogan Onugoren M, Golombeck KS, Bien C et al. Immunoadsorption therapy in autoimmune encephalitides. Neurol Neuroimmunol Neuroinflammation 2016; 3: e207

[49] Gastaldi M, Thouin A, Vincent A. Antibody-mediated autoimmune encephalopathies and immunotherapies. Neurotherapeutics 2016; 13: $147-162$

[50] Tatencloux S, Chretien P, Rogemond V et al. Intrathecal treatment of anti-N-Methyl-D-aspartate receptor encephalitis in children. Dev Med Child Neurol 2015; 57: 95-99

[51] Behrendt V, Krogias C, Reinacher-Schick A et al. Bortezomib treatment for patients with anti- $\mathrm{N}$-methyl-d-aspartate receptor encephalitis. JAMA Neurol 2016; 73: 1251-1253

[52] Scheibe F, Prüss $\mathrm{H}$, Mengel AM et al. Bortezomib for treatment of therapy-refractory anti-NMDA receptor encephalitis. Neurology 2017; 88: 366-370

[53] Gresa-Arribas N, Titulaer M], Torrents A et al. Antibody titres at diagnosis and during follow-up of anti-NMDA receptor encephalitis: A retrospective study. Lancet Neurol 2014; 13: 167-177

[54] Irani SR, Alexander S, Waters $P$ et al. Antibodies to Kv1 potassium channel-complex proteins leucine-rich, glioma inactivated 1 protein and contactin-associated protein- 2 in limbic encephalitis, Morvan's syndrome and acquired neuromyotonia. Brain 2010; 133: 2734-2748

[55] Lai M, Huijbers MGM, Lancaster E et al. Investigation of LGI1 as the antigen in limbic encephalitis previously attributed to potassium channels: a case series. Lancet Neurol 2010; 9: 776-785

[56] Lancaster E, Huijbers MGM, Bar V et al. Investigations of caspr2, an autoantigen of encephalitis and neuromyotonia. Ann Neurol 2011; 69: $303-311$
[57] Irani SR, Michell AW, Lang B et al. Faciobrachial dystonic seizures precede Lgi1 antibody limbic encephalitis. Ann Neurol 2011; 69: 892-900

[58] van Sonderen A, Ariño H, Petit-Pedrol M et al. The clinical spectrum of Caspr2 antibody-associated disease. Neurology 2016; 87: 521-528

[59] Lang B, Makuch M, Moloney T et al. Intracellular and non-neuronal targets of voltage-gated potassium channel complex antibodies. J Neurol Neurosurg Psychiatry. 88: 353-361

[60] van Sonderen A, Thijs RD, Coenders EC et al. Anti-LGI1 encephalitis: Clinical syndrome and long-term follow-up. Neurology 2016; 87: 1449-1456

[61] Ohkawa T, Fukata Y, Yamasaki M et al. Autoantibodies to epilepsyrelated LGI1 in limbic encephalitis neutralize LGI1-ADAM22 interaction and reduce synaptic AMPA receptors. J Neurosci 2013; 33: 18161-18174

[62] Malter MP, Frisch C, Schoene-Bake JC et al. Outcome of limbic encephalitis with VGKC-complex antibodies: Relation to antigenic specificity. J Neurol 2014; 261: 1695-1705

[63] Thieben M], Lennon VA, Boeve BF et al. Potentially reversible autoimmune limbic encephalitis with neuronal potassium channel antibody. Neurology 2004; 62: 1177-1182

[64] Shin Y-W, Lee S-T, Shin J-W et al. VGKC-complex/LGI1-antibody encephalitis: clinical manifestations and response to immunotherapy. J Neuroimmunol 2013; 265: 75-81

[65] Wong SH, Saunders MD, Larner A] et al. An effective immunotherapy regimen for VGKC antibody-positive limbic encephalitis. J Neurol Neurosurg Psychiatry 2010; 81: 1167-1169

[66] Vincent A, Buckley C, Schott JM et al. Potassium channel antibodyassociated encephalopathy: A potentially immunotherapy-responsive form of limbic encephalitis. Brain 2004; 127: 701-712

[67] Irani SR, Stagg C], Schott JM et al. Faciobrachial dystonic seizures: the influence of immunotherapy on seizure control and prevention of cognitive impairment in a broadening phenotype. Brain 2013; 136: 3151-3162

[68] Tobin WO, Lennon VA, Komorowski L et al. DPPX potassium channel antibody. Neurology 2014; 83: 1797-1803

[69] Lai M, Hughes EG, Peng X et al. AMPA receptor antibodies in limbic encephalitis alter synaptic receptor location. Ann Neurol 2009; 65: 424-434

[70] Lancaster E, Lai M, Peng X et al. Antibodies to the GABA(B) receptor in limbic encephalitis with seizures: Case series and characterisation of the antigen. Lancet Neurol 2010; 9: 67-76

[71] Sabater L, Gaig C, Gelpi E et al. A novel non-rapid-eye movement and rapid-eye-movement parasomnia with sleep breathing disorder associated with antibodies to IgLON5: A case series, characterisation of the antigen, and post-mortem study. Lancet Neurol 2014; 13 : 575-586

[72] Gorman MP. Update on diagnosis, treatment, and prognosis in opsoclonus-myoclonus-ataxia syndrome. Curr Opin Pediatr 2010; 22: $745-750$

[73] Vedeler CA, Antoine JC, Giometto B et al. Management of paraneoplastic neurological syndromes: Report of an EFNS Task Force. Eur J Neurol 2006; 13: 682-690

[74] Pranzatelli MR, Tate ED, Swan JA et al. B cell depletion therapy for new-onset opsoclonus-myoclonus. Mov Disord 2010; 25: 238-242

[75] Mitchell WG, Wooten AA, O'Neil SH et al. Effect of Increased immunosuppression on developmental outcome of opsoclonus myoclonus syndrome (OMS). J Child Neurol 2015; 30: 976-982

[76] Armangué T, Sabater L, Torres-Vega E et al. Clinical and Immunological Features of opsoclonus-myoclonus syndrome in the era of neuronal cell surface antibodies. JAMA Neurol 2016; 73: 417-424 
[77] Bataller L, Graus F, Saiz A et al. Clinical outcome in adult onset idiopathic or paraneoplastic opsoclonus-myoclonus. Brain J Neurol 2001; 124: 437-443

[78] Armangue T, Titulaer M], Sabater L et al. A novel treatment-responsive encephalitis with frequent opsoclonus and teratoma. Ann Neurol 2014; 75: 435-441

[79] Glatz K, Meinck H, Wildemann B. Parainfectious opsoclonusmyoclonus syndrome: high dose intravenous immunoglobulins are effective. J Neurol Neurosurg Psychiatry 2003; 74: 279-280

[80] Murinson BB, Guarnaccia JB. Stiff-person syndrome with amphiphysin antibodies. Neurology 2008; 71: 1955-1958

[81] McKeon A, Robinson MT, McEvoy KM et al. Stiff-man syndrome and variants: clinical course, treatments, and outcomes. Arch Neurol 2012; 69: 230-238

[82] Carvajal-González A, Leite MI, Waters P et al. Glycine receptor antibodies in PERM and related syndromes: Characteristics, clinical features and outcomes. Brain 2014; 137: 2178-2192

[83] Spehlmann R, Norcross K, Rasmus SC et al. Improvement of stiff-man syndrome with sodium valproate. Neurology 1981; 31: 1162-1163

[84] Sechi G, Barrocu M, Piluzza MG et al. Levetiracetam in stiff-person syndrome. J Neurol 2008; 255: 1721-1725

[85] Holmøy T. Long-term effect of gabapentin in stiff limb syndrome: A case report. Eur Neurol 2007; 58: 251-252

[86] Stayer C, Tronnier V, Dressnandt J et al. Intrathecal baclofen therapy for stiff-man syndrome and progressive encephalomyelopathy with rigidity and myoclonus. Neurology 1997; 49: 1591-1597

[87] Schmierer K, Valdueza JM, Bender A et al. Atypical stiff-person syndrome with spinal MRI findings, amphiphysin autoantibodies, and immunosuppression. Neurology 1998; 51: 250-252

[88] Faissner S, Lukas C, Reinacher-Schick A et al. Amphiphysin-positive paraneoplastic myelitis and stiff-person syndrome. Neurol Neuroimmunol Neuroinflamm 2016; 3: e285

[89] Sommer C, Weishaupt A, Brinkhoff J et al. Paraneoplastic stiff-person syndrome: Passive transfer to rats by means of $\mathrm{IgG}$ antibodies to amphiphysin. Lancet 2005; 365: 1406-1411

[90] Dalakas MC, Fujii M, Li M et al. High-dose intravenous immune globulin for stiff-person syndrome. N Engl J Med 2001; 345: 1870-1876

[91] Stiff-Man-Syndrom. Leitlinie der Deutschen Gesellschaft für Neurologie. Im Internet: www.dgn.org Stand: Sep. 2012

[92] Pagano MB, Murinson BB, Tobian AAR et al. Efficacy of therapeutic plasma exchange for treatment of stiff-person syndrome. Transfusion (Paris) 2014; 54: 1851-1856

[93] Baker M, Das M, Isaacs J et al. Treatment of stiff person syndrome with rituximab. J Neurol Neurosurg Psychiatry 2005; 76: 999-1001

[94] Flanagan EP, McKeon A, Lennon VA et al. Paraneoplastic isolated myelopathy: Clinical course and neuroimaging clues. Neurology 2011; 76: 2089-2095

[95] Pittock S], Lennon VA. Aquaporin-4 autoantibodies in a paraneoplastic context. Arch Neurol 2008; 65: 629-632

[96] Figueroa M, Guo Y, Tselis A et al. Paraneoplastic neuromyelitis optica spectrum disorder associated with metastatic carcinoid expressing aquaporin-4. JAMA Neurol 2014; 71: 495-498

[97] Leypoldt F, Eichhorn P, Saager C et al. Successful immunosuppressive treatment and long-term follow-up of anti-Ri-associated paraneoplastic myelitis. J Neurol Neurosurg Psychiatry 2006; 77: 1199-1200

[98] Rajabally YA, Qaddoura B, Abbott RJ. Steroid-responsive paraneoplastic demyelinating neuropathy and myelopathy associated with breast carcinoma. J Clin Neuromuscul Dis 2008; 10: 65-69
[99] Klein C]. Autoimmune-mediated peripheral neuropathies and autoimmune pain. Handb Clin Neurol 2016; 133: 417-446

[100] McKeon A, Lennon VA, Lachance DH et al. Ganglionic acetylcholine receptor autoantibody. Arch Neurol 2009; 66: 735-741

[101] lodice V, Kimpinski K, Vernino S et al. Efficacy of immunotherapy in seropositive and seronegative putative autoimmune autonomic ganglionopathy. Neurology 2009; 72: 2002-2008

[102] Titulaer MJ, Lang B, Verschuuren J]. Lambert-Eaton myasthenic syndrome: From clinical characteristics to therapeutic strategies. Lancet Neurol 2011; 10: 1098-1107

[103] Diagnostik und Therapie der Myasthenia gravis und des LambertEaton-Syndroms. Leitlinie der Deutschen Gesellschaft für Neurologie. Im Internet: www.dgn.org Stand: Sep. 2014

[104] Wang J, Guo G, Chen G et al. Meta-analysis of the association of dermatomyositis and polymyositis with cancer. $\mathrm{Br}$ J Dermatol 2013; 169: $838-847$

[105] Chinoy H, Fertig N, Oddis CV et al. The diagnostic utility of myositis autoantibody testing for predicting the risk of cancer-associated myositis. Ann Rheum Dis 2007; 66: 1345-1349

[106] Rozelle A, Trieu S, Chung L. Malignancy in the setting of the anti-synthetase syndrome. J Clin Rheumatol Pract Rep Rheum Musculoskelet Dis 2008; 14: 285-288

[107] Hill CL, Zhang Y, Sigurgeirsson B et al. Frequency of specific cancer types in dermatomyositis and polymyositis: A population-based study. Lancet 2001; 357: 96-100

[108] Suber TL, Casciola-Rosen L, Rosen A. Mechanisms of disease: Autoantigens as clues to the pathogenesis of myositis. Nat Clin Pract Rheumatol 2008; 4: 201-209

[109] Dankó K, Ponyi A, Molnar AP et al. Paraneoplastic myopathy. Curr Opin Rheumatol 2009; 21: 594-598

[110] Myositissyndrome, Leitlinien der Deutschen Gesellschaft für Neurologie. Im Internet: www.dgn.org Stand: Sep. 2014

[111] Khosroshahi A, Carruthers MN, Deshpande V et al. Rituximab for the treatment of IgG4-related disease: Lessons from 10 consecutive patients. Medicine (Baltimore) 2012; 91: 57-66

[112] Gomez AM, Willcox N, Molenaar PC et al. Targeting plasma cells with proteasome inhibitors: Possible roles in treating myasthenia gravis? Ann N Y Acad Sci 2012; 1274: 48-59

[113] Tanaka T, Narazaki M, Kishimoto T. Anti-interleukin-6 receptor antibody, tocilizumab, for the treatment of autoimmune diseases. FEBS Lett 2011; 585: 3699-3709

[114] Ayzenberg I, Kleiter I, Schröder A et al. Interleukin 6 receptor blockade in patients with neuromyelitis optica nonresponsive to anti-CD20 therapy. JAMA Neurol 2013; 70: 394-397

[115] Krogias C, Hoepner R, Müller A et al. Successful treatment of anti-Caspr2 syndrome by interleukin 6 receptor blockade through tocilizumab. JAMA Neurol 2013; 70: 1056-1059

[116] Ringelstein M, Ayzenberg I, Harmel J et al. Long-term therapy with interleukin 6 receptor blockade in highly active neuromyelitis optica spectrum disorder. JAMA Neurol 2015; 72: 756-763

[117] Orange D, Frank M, Tian S et al. Cellular immune suppression in paraneoplastic neurologic syndromes targeting intracellular antigens. Arch Neurol 2012; 69: 1132-1140

[118] Bertrand A, Kostine M, Barnetche T et al. Immune related adverse events associated with anti-CTLA-4 antibodies: Systematic review and meta-analysis. BMC Med 2015; 13: 211

[119] Williams T], Benavides DR, Patrice K-A et al. Association of autoimmune encephalitis with combined immune checkpoint inhibitor treatment for metastatic cancer. JAMA Neurol 2016; 73: 928-933 